\title{
Olaratumab and doxorubicin for the treatment of metastatic soft tissue sarcoma: a retrospective case series
}

\author{
Joon Young Hur ${ }^{1}$, Se Hoon Park ${ }^{1}$, Su Jin Lee ${ }^{1,2}$ \\ ${ }^{1}$ Division of Hematology and Oncology, Department of Medicine, Samsung Medical Center, Sungkyunkwan University School of \\ Medicine, Seoul, Korea \\ ${ }^{2}$ Division of Hematology and Oncology, Department of Internal Medicine, Ewha Womans University School of Medicine, Seoul, \\ Korea
}

Received: March 8, 2019

Revised: April 1, 2019

Accepted: April 18, 2019

Corresponding author:

Su Jin Lee

Division of Hematology and

Oncology, Department of

Medicine, Samsung Medical

Center, Sungkyunkwan

University School of Medicine,

81 Irwon-ro, Gangnam-gu,

Seoul 06351, Korea

Tel: +82-2-3410-3459

E-mail: sujinlee421@gmail.com

This is an Open Access article distributed under the terms of the Creative Commons Attribution Non-Commercial License (http:// creativecommons.org/licenses/ by-nc/4.0/).

\section{ABSTRACT}

Purpose: Soft tissue sarcomas (STS) are a rare and heterogeneous tumor group with limited treatment options. This study aimed to evaluate the anti-tumor efficacy of olaratumab and doxorubicin in patients with advanced STS in front-line and salvage setting.

Methods: Patients with STS who received olaratumab and doxorubicin between October 2017 and August 2018 were retrospectively reviewed. Response rate, progression-free survival (PFS), and overall survival (OS) were analyzed according to histologic subtype, Eastern Cooperative Oncology Group performance status, and number of prior chemotherapy regimens.

Results: A total of 26 patients were included in the analysis. The common histologic subtypes included undifferentiated/unclassified sarcoma $(n=8)$, leiomyosarcoma $(n=7)$, liposarcoma $(n=3)$, and solitary fibrous tumor $(n=2)$. Of 26 patients, 10 patients (38.4\%) received more than two chemotherapy regimens before olaratumab and doxorubicin. At the time of analysis, 152 cycles of olaratumab had been administered (median, five cycles), and there was no treatment-related mortality. The disease control rate was $61.5 \%(n=16)$, and the overall response rate was $15.3 \%$ (partial response, $n=4$; complete response, $n=0$ ). Partial responses were achieved in one patient with solitary fibrous tumor, one with dedifferentiated liposarcoma, one with leiomyosarcoma, and one with pleomorphic rhabdomyosarcoma, all in salvage setting. With a median follow-up duration of 11.1 months ( $95 \%$ confidence interval [Cl], 11.1 to 13.1 months), the median PFS was 4.1 months ( $95 \% \mathrm{Cl}, 2.1$ to 6.1 months), and the median OS was not reached.

Conclusion: Olaratumab and doxorubicin demonstrated acceptable anti-tumor activity in Asian patients with sarcoma.

Keywords: Doxorubicin; Olaratumab; Sarcoma 


\section{INTRODUCTION}

Soft tissue sarcomas (STS) account for $6 \%$ of childhood cancers and $1 \%$ of all adult malignancies [1,2]. Among more than 100 subtypes of STS, the most common histologic subtypes are undifferentiated unclassified sarcoma, liposarcoma, leiomyosarcoma, synovial sarcoma and malignant peripheral nerve sheath tumors [3]. Surgical resection with appropriately negative margins is the standard primary treatment for resectable STS, and patients with extremity STS are commonly treated with limb preservation surgery in combination with adjuvant radiotherapy [4]. Chemotherapy with single agents (e.g., dacarbazine, doxorubicin, epirubicin, ifosfamide, pazopanib, trabectedin, or eribulin) or anthracycline-based combination regimens (doxorubicin or epirubicin with ifosfamide and/or dacarbazine) has been widely used for patients with advanced, unresectable, or metastatic disease [5-8].

Olaratumab (Lartruvo, Eli Lilly and Company, Indianapolis, IN, USA) is a fully human immunoglobulin G1 monoclonal antibody that selectively binds the external domain of human platelet-derived growth factor receptor- $\alpha$ (PDGFR) with high affinity and blocks ligand binding [2]. In October 2016, the U.S. Food and Drug Administration granted accelerated approval to olaratumab in combination with doxorubicin (olaratumab+doxorubicin) for the treatment of adult patients with STS with a histologic subtype for which an anthracycline-containing regimen is appropriate and not amenable to curative treatment with radiotherapy or surgery [9].

However, studies on the efficacy and tolerability of olaratumab and doxorubicin in Asian patients with sarcoma are limited. In addition, clinical outcome and tolerability of the regimen in heavily pre-treated patients are scarcely reported. Hence, the efficacy and tolerability of olaratumab and doxorubicin in Asian patients is yet to be established and should be evaluated. Therefore, this retrospective study aimed to evaluate the anti-tumor efficacy of olaratumab and doxorubicin in STS patients in Asia. Particularly, we focused on evaluating the tolerability and anti-tumor efficacy in heavily pre-treated refractory sarcoma patients.

\section{METHODS}

\section{Study design}

We retrospectively reviewed the medical records of patients with advanced STS who were treated with olaratumab and doxorubicin between October 2017 and August 2018 at Samsung Medical Center (SMC), Korea. Inclusion criteria were as follows: (1) histologically confirmed diagnosis of advanced unresectable or metastatic STS not amenable to curative treatment with surgery or radiotherapy and (2) availability of complete clinical information including patient demographics, primary tumor site, stage, and treatment record. The following clinicopathologic variables were collected: age, sex, histologic type, extent of metastasis, Eastern Cooperative Oncology Group (ECOG) performance status, and treatment history. The study was reviewed and approved by the Institutional Review Board of SMC (IRB No. 2018-08-107), and informed consent was waived.

\section{Treatment}

Patients receive olaratumab $(15 \mathrm{mg} / \mathrm{kg})$ intravenously on days 1 and 8 plus doxorubicin $\left(75 \mathrm{mg} / \mathrm{m}^{2}\right)$ on day 1 of each 21-day cycle. After eight cycles, patients were allowed to receive olaratumab monotherapy until disease progression provided that they have no disease progression or unacceptable toxicities. During cycles 5 to 8 , dexrazoxane was allowed on day 1 of each cycle to reduce the potential for doxorubicin-related cardiotoxicity. Cardiac echography was done for monitoring cardiac function during treatment. Treatment was continued until disease progression, unacceptable toxicity, or patient refusal. Tumor response was evaluated every 6 or 9 weeks using computed tomography scans or magnetic resonance imaging. Responses were assessed according to the Response Evaluation Criteria in Solid Tumors (RECIST), version 1.1. Adverse events were graded according to the $\mathrm{Na}$ tional Cancer Institute Common Terminology Criteria for Adverse Events version 4.0.

\section{Statistical analysis}

Standard descriptive and analytical methods were used to describe the patient population and their baseline characteristics. Overall survival (OS) was defined as the time from the initiation of olaratumab and doxorubicin treatment to the date of death or last follow-up. Progression-free survival (PFS) was defined as the time from initiation of olaratumab and doxorubicin treatment to the date of documented disease progression or death from any cause. Kaplan-Meier curves were used to analyze time-to-event variables, and 95\% confidence intervals ( $\mathrm{Cls}$ ) were computed for time-toevent medians. Survival comparisons were performed using univariate log-rank tests. All statistical analyses were performed using R version 3.4.4 (R Foundation for Statistical Computing, Vienna, Austria), and two-tailed P-values of less than 0.05 were considered statistically significant. 


\section{RESULTS}

\section{Patient characteristics}

Between October 2017 and August 2018, 26 STS patients received olaratumab and doxorubicin. Baseline patient characteristics at the start of olaratumab and doxorubicin treatment are summarized in Table 1 . The median age was 53 years (range, 25 to 73 years), and $57.6 \%$ of patients were male. Most patients (92.3\%) had a good ECOG performance status (0-1). The distribution of histologic subtypes was as follows: eight patients had undifferentiated/unclassified sarcoma; seven, leiomyosarcoma; three, liposarcoma; three, pleomorphic sarcoma; two, solitary fibrous tumor. French Fédération Nationale des Centres de Lutte Contre le Cancer grades were available for eight patients (30.6\%); six of these patients $(23.0 \%)$ had grade 3 sarcoma. The primary tumor site was the trunk or retroperitoneum in 19 patients (73.0\%) and the extremities in five patients (19.2\%). The most common metastasis sites were the lung $(n=14,53.8 \%)$, liver $(n=7,26.9 \%)$, and bone $(n=4,15.3 \%)$.

A total of 13 patients (50\%) of patients previously had at least one chemotherapy regimen before olaratumab and doxorubicin treatment, while the other 13 patients were chemo-naïve. Ten patients (38.4\%) were heavily pre-treated with two or more lines of chemotherapy.

A total of nine (34.6\%), eight (30.7\%), seven (26.9\%), and two patients $(7.6 \%)$ had received prior pazopanib chemotherapy; docetaxel and gemcitabine chemotherapy; etoposide, ifosfamide, and cisplatin chemotherapy, and pembrolizumab chemotherapy, respectively.

A total of 152 cycles of olaratumab and doxorubicin therapy were administered, with a median of five cycles per patient (range, 1 to 16 cycles). At the time of analysis, three patients were still receiving olaratumab and doxorubicin treatment, and the remaining 23 patients had stopped treatment because of progression $(n=21)$, patient refusal $(n=1)$, or other reasons $(n=1)$.

\section{Tumor responses}

Of the 26 patients, 25 were evaluable for tumor response except for one patient follow-up loss. No patient achieved a complete response, but four patients achieved partial responses, yielding a $15.3 \%(95 \% \mathrm{Cl}, 1.46 \%$ to $29.13 \%)$ overall response rate. Partial responses were achieved in one patient with solitary fibrous tumor, one with dedifferentiated liposarcoma, one with leiomyosarcoma, and one with pleomorphic rhabdomyosarcoma. Of the four patients with par-
Table 1. Patient demographics and clinical characteristics

\begin{tabular}{|c|c|}
\hline Characteristic & Value \\
\hline Total & $26(100)$ \\
\hline Age (yr) & $53(25-73)$ \\
\hline \multicolumn{2}{|l|}{ Sex } \\
\hline Male & $15(57.6)$ \\
\hline Female & $11(42.3)$ \\
\hline \multicolumn{2}{|l|}{ ECOG PS } \\
\hline 1 & $24(92.3)$ \\
\hline 2 & $2(7.6)$ \\
\hline \multicolumn{2}{|l|}{ Histology } \\
\hline Undifferentiated/unclassified sarcoma & $8(30.7)$ \\
\hline Leiomyosarcoma & $7(26.9)$ \\
\hline Liposarcoma & $3(11.5)$ \\
\hline Solitary fibrous tumor & $2(7.6)$ \\
\hline Pleomorphic rhabdomyosarcoma & $1(3.8)$ \\
\hline Endometrial stromal sarcoma & $1(3.8)$ \\
\hline Epithelioid sarcoma & $1(3.8)$ \\
\hline Carcinosarcoma & $1(3.8)$ \\
\hline Clear cell sarcoma & $1(3.8)$ \\
\hline MPNST & $1(3.8)$ \\
\hline \multicolumn{2}{|l|}{ FNCLCC grade } \\
\hline 1 & 0 \\
\hline 2 & $2(7.6)$ \\
\hline 3 & $6(23.0)$ \\
\hline Unknown & $18(69.2)$ \\
\hline \multicolumn{2}{|l|}{ Primary site } \\
\hline Trunk or retroperitoneum & $19(73.0)$ \\
\hline Extremity & $5(19.2)$ \\
\hline Head and neck & $2(7.6)$ \\
\hline \multicolumn{2}{|l|}{ Organ involvement } \\
\hline Lung & $14(53.8)$ \\
\hline Liver & $7(26.9)$ \\
\hline Bone & $4(15.3)$ \\
\hline \multicolumn{2}{|c|}{ No. of prior chemotherapy regimens chemotherapies } \\
\hline 0 & $13(50.0)$ \\
\hline 1 & $3(11.5)$ \\
\hline$\geq 2$ & $10(38.4)$ \\
\hline \multicolumn{2}{|l|}{ Agents used in prior regimen(s) } \\
\hline Pazopanib & $9(34.6)$ \\
\hline Docetaxel/gemcitabine & $8(30.7)$ \\
\hline VIP & $7(26.9)$ \\
\hline Pembrolizumab & $2(7.6)$ \\
\hline Nivolumab & $1(3.8)$ \\
\hline
\end{tabular}

Values are presented as number (\%) or median (range). ECOG, Eastern Cooperative Oncology Group; PS, performance status; MPNST, malignant peripheral nerve sheath tumor; FNCLCC, Fédération Nationale des Centres de Lutte Contre Le Cancer; VIP, etoposide, ifosfamide, and cisplatin. 
tial responses, one maintained treatment response for more than 13 cycles at the time of this writing. Twelve patients
(46.1\%) achieved stable disease, yielding a disease control rate of $61.5 \%(95 \% \mathrm{Cl}, 42.79 \%$ to $80.20 \%)$.
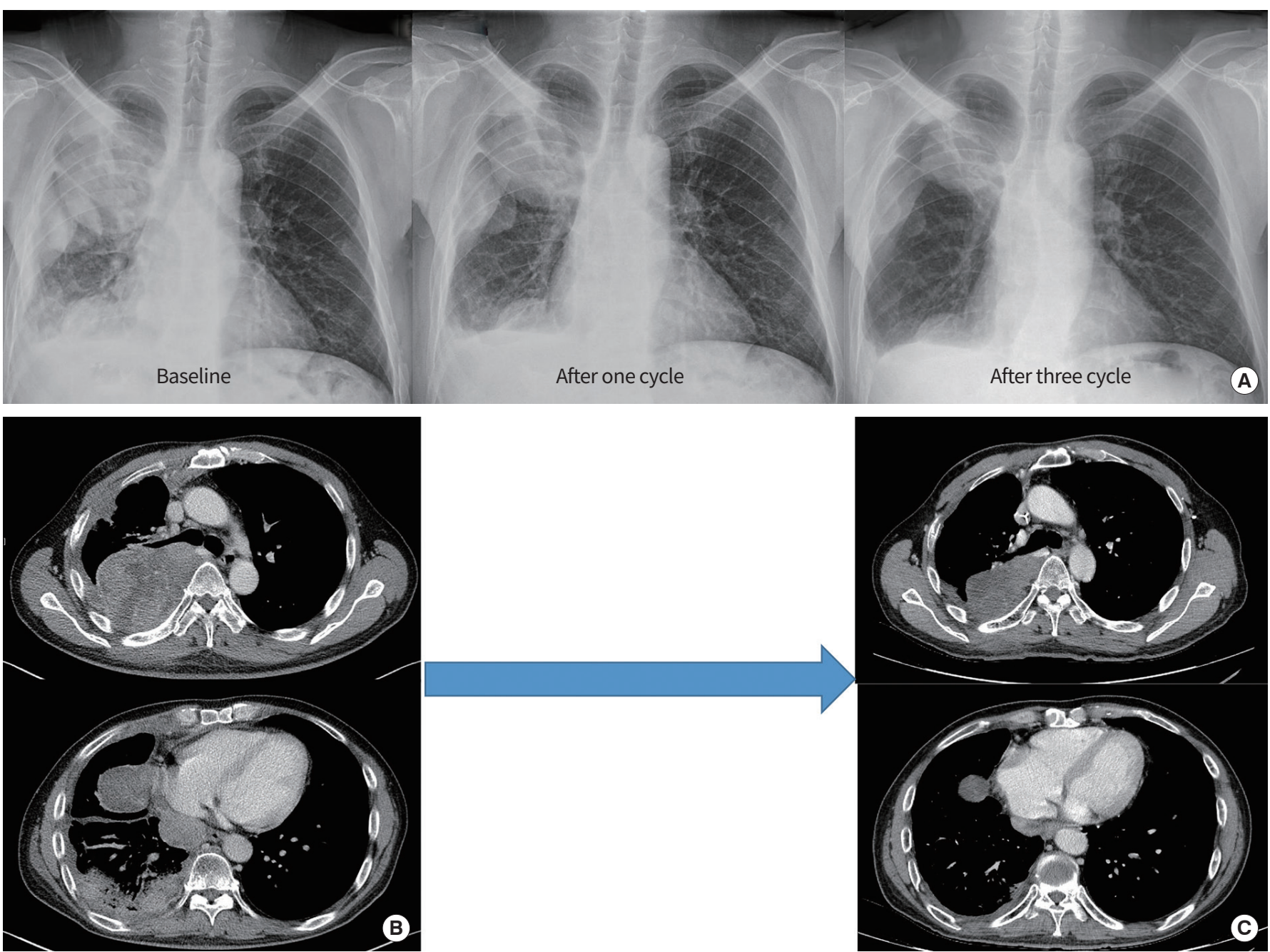

Fig. 1. One case of partial response. (A) Forty-five-year-old male patient with dedifferentiated liposarcoma originated from retroperitoneum had disseminated lung metastasis with extensive pleural seeding at baseline. (B) After one cycle of doxorubicin, olaratumab therapy, multiple pleural masses decreased in chest X-ray. (C) After three cycles of therapy, he achieved partial response and continues to respond to olaratumab/doxorubicin for 5 months.
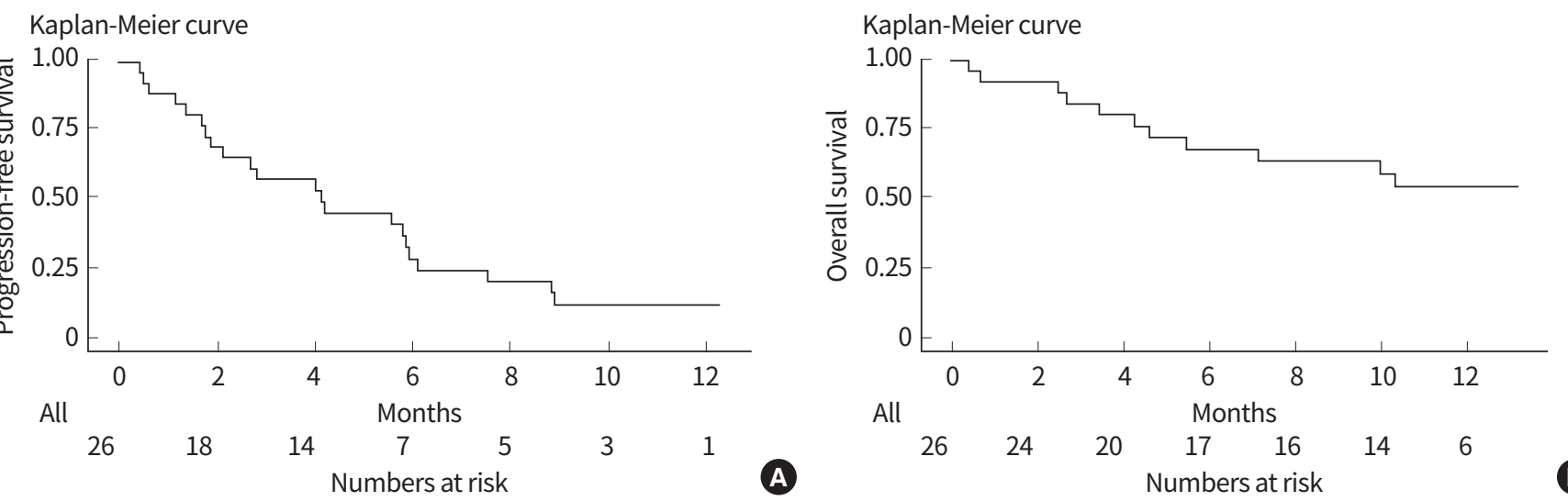

Fig. 2. Analysis of survival probability. (A) Progression-free survival and (B) overall survival. 
Notably, a 45-year-old male patient with dedifferentiated liposarcoma from the retroperitoneum had rapidly progressive disease. He underwent prior chemotherapy including adriamycin and cisplatin, docetaxel and gemcitabine, pazopanib, paclitaxel, etoposide and cisplatin, doxorubicin, ifosfamide, and dacarbazine, and pembrolizumab. However, he was alive for 7 years at the time of this writing owing to olaratumab and doxorubicin with partial response. At the time of olaratumab/doxorubicin chemotherapy, the patient had disseminated lung metastases with extensive pleural seeding. However, after three cycles of olaratumab/doxorubicin, the patient achieved partial response and continues to respond

Table 2. Survival of patients with various histologic subtypes

\begin{tabular}{lccc}
\hline Histology & No. $(\%)$ & PFS (mo) $(95 \% \mathrm{Cl})$ & $\mathrm{OS}(\mathrm{mo})(95 \% \mathrm{Cl})$ \\
\hline Undifferentiated/unclassified sarcoma & $8(30.7)$ & $2.2(1.7-\mathrm{NA})$ & $\mathrm{NR}$ \\
Leiomyosarcoma & $7(26.9)$ & $4.0(1.3-\mathrm{NA})$ & $\mathrm{NR}$ \\
Liposarcoma & $3(11.5)$ & $\mathrm{NR}$ & $\mathrm{NR}$ \\
Solitary fibrous tumor & $2(7.6)$ & $\mathrm{NA}$ & $\mathrm{NA}$ \\
Pleomorphic rhabdomyosarcoma & $1(3.8)$ & $\mathrm{NA}$ & $\mathrm{NA}$ \\
Endometrial stromal sarcoma & $1(3.8)$ & $\mathrm{NA}$ & $\mathrm{NA}$ \\
Epithelioid sarcoma & $1(3.8)$ & $\mathrm{NA}$ & $\mathrm{NA}$ \\
Carcinosarcoma & $1(3.8)$ & $\mathrm{NA}$ & $\mathrm{NA}$ \\
Clear cell sarcoma & $1(3.8)$ & $\mathrm{NA}$ & $\mathrm{NA}$ \\
MPNST & $1(3.8)$ & $\mathrm{NA}$ \\
Total & $26(100)$ & $\mathrm{NR}$ \\
\hline
\end{tabular}

PFS, progression-free survival; $\mathrm{Cl}$, confidence interval; OS, overall survival; NA, not available; NR, not reached; MPNST, malignant peripheral nerve sheath tumor.

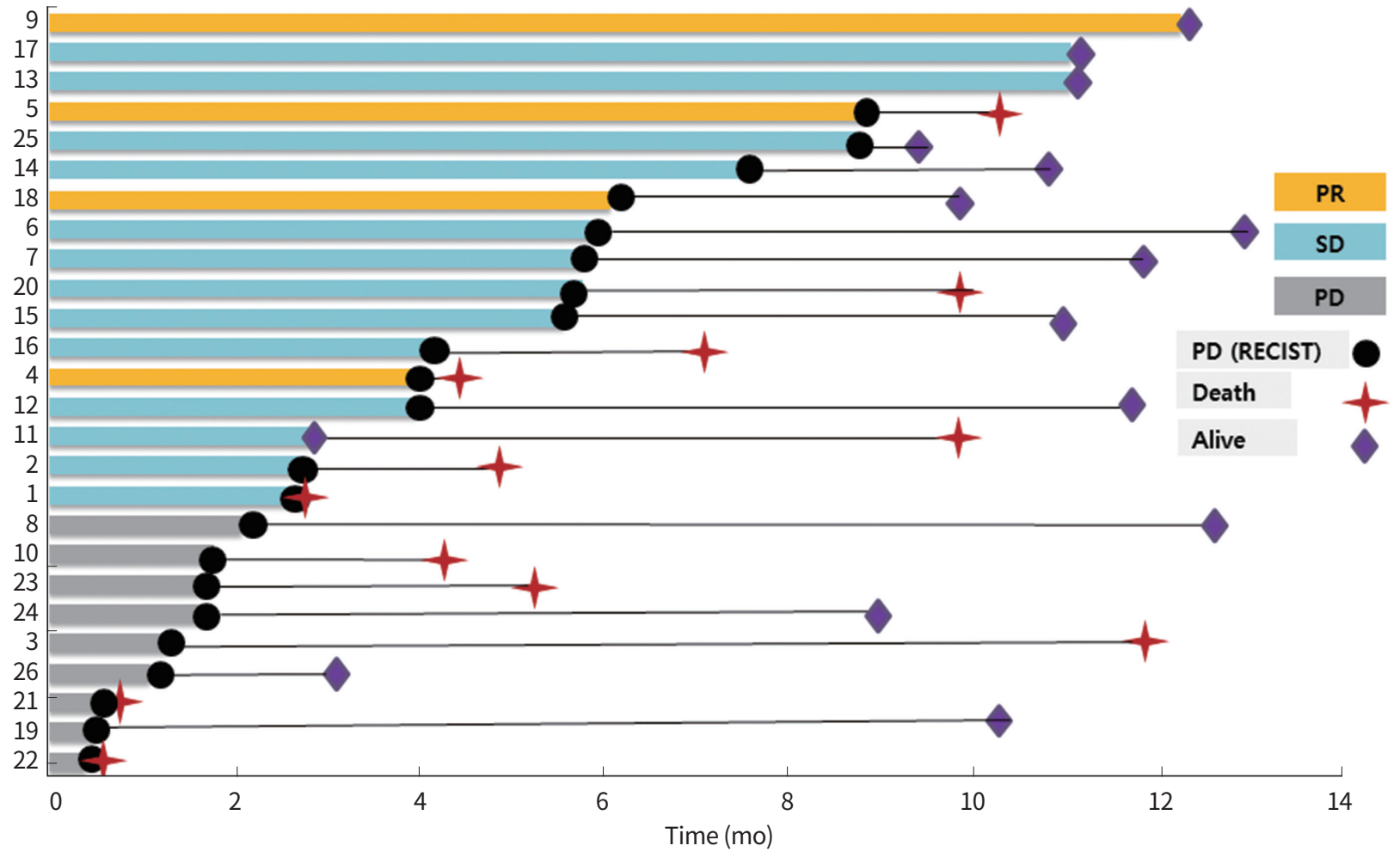

Fig. 3. Swimmer's plot of each patient. PR, partial response; SD, stable disease; PD, progressive disease; RECIST, response evaluation criteria in solid tumors. 
PRECISION AND FUTURE MIEDICINE

Olaratumab and doxorubicin in soft tissue sarcoma

Table 3. Treatment-related toxicity

\begin{tabular}{|c|c|c|c|c|}
\hline \multirow{2}{*}{ Variable } & \multicolumn{4}{|c|}{ Toxicity no. (\%) } \\
\hline & Grade 1 & Grade 2 & Grade 3 & Grade 4 \\
\hline \multicolumn{5}{|l|}{ Hematologic toxicity } \\
\hline Anemia & $1(3.8)$ & $1(3.8)$ & $1(3.8)$ & 0 \\
\hline Neutropenia & 0 & $4(15.3)$ & $7(26.9)$ & 0 \\
\hline Febrile neutropenia & 0 & 0 & $4(15.3)$ & 0 \\
\hline Thrombocytopenia & $2(7.6)$ & 0 & 0 & 0 \\
\hline Non-hematologic toxicity & & & & 0 \\
\hline Nausea & $1(3.8)$ & $2(7.6)$ & 0 & 0 \\
\hline Constipation & $1(3.8)$ & 0 & 0 & 0 \\
\hline Mucositis & 0 & $2(7.6)$ & 0 & 0 \\
\hline Anorexia & $5(19.2)$ & $1(3.8)$ & 0 & 0 \\
\hline Diarrhea & 0 & $1(3.8)$ & 0 & 0 \\
\hline Fatigue & $1(3.8)$ & $2(7.6)$ & 0 & 0 \\
\hline Skin rash & $1(3.8)$ & 0 & 0 & 0 \\
\hline
\end{tabular}

to olaratumab/doxorubicin for 5 months at the time of this writing (Fig. 1). The cumulative dose of doxorubicin was 355 $\mathrm{mg}$ and there was no doxorubicin-related cardiotoxicity.

\section{Survival outcomes}

With a median follow-up of 11.1 months $(95 \% \mathrm{Cl}, 11.1$ to 13.1 months), the median PFS was 4.1 months ( $95 \% \mathrm{Cl}, 2.1$ to 6.1 months), and the median OS was not reached (Fig. 2). We analyzed the PFS and OS in patients according to different histologic subtypes (Table 2). In patients with leiomyosarcoma, the median PFS was 4.0 months $(95 \% \mathrm{Cl}, 1.3$ to not available months), and the median OS was not reached. Meanwhile, the median PFS in patients with undifferentiated/unclassified sarcoma was 2.0 months $(95 \% \mathrm{Cl}, 1.7$ to not available). Each patient's treatment response and survival was shown in Fig. 3.

Age ( $>50$ years vs. $\leq 50$ years, $P=0.089$ ), sex (male vs. female, $\mathrm{P}=0.169)$, and number of prior chemotherapy regimens ( $>2$ vs. $\leq 1, P=0.098$ ) were not significantly correlated with PFS at univariate level. However, performance status (ECOG 2 vs. 1) was a significant adverse factor of PFS for olaratumab/doxorubicin treatment $(P=0.001)$.

\section{Treatment-related toxicity}

As shown in Table 3, the most common non-hematologic toxicities were anorexia (grade $1-2, n=6$ ), nausea (grade $1-2$, $n=3$ ), mucositis (grade $2, n=2$ ), fatigue (grade $1-2, n=3$ ), constipation (grade 1, $n=1$ ), diarrhea (grade $2, n=1$ ), and skin rash (grade 1, $n=1$ ). Grade 3 hematologic toxicities were neutropenia $(n=7)$, febrile neutropenia $(n=4)$, and anemia $(n=1)$. There was no treatment-related mortality associated with olaratumab and doxorubicin treatment both in frontline or salvage settings. Most non-hematologic toxicities were reversible with appropriate medical management (e.g., loperamide for diarrhea) and dose reduction. The dose of olaratumab was reduced in $15.3 \%$ of patients $(n=4)$ due to treatment-related toxicities.

\section{DISCUSSION}

We demonstrated that olaratumab and doxorubicin are a feasible treatment option with acceptable anti-tumor activity in metastatic STS patients. Moreover, the regimen was relatively safe and efficacious in heavily pre-treated patients with metastatic sarcoma. Of note, we observed that olaratumab and doxorubicin tended to have better anti-tumor activity in patients with leiomyosarcoma, but less activity in patients with undifferentiated/unclassified sarcoma.

Two open-label dose-escalation phase I studies evaluated olaratumab as a single agent in patients with advanced solid tumors [10]. The combination of olaratumab plus doxorubicin has provided a new front-line therapeutic option for STS patients. An open-label phase Ib and randomized phase II trial of patients with advanced STS demonstrated that the addition of olaratumab to doxorubicin prolonged PFS by 2.5 months and $O S$ by 11.8 months than doxorubicin alone [11]. 
The recently reported results of A Study of Doxorubicin Plus Olaratumab (LY3012207) in Participants With Advanced or Metastatic Soft Tissue Sarcoma (ANNOUNCE), the phase III study of olaratumab in combination with doxorubicin in patients with advanced or metastatic soft-tissue sarcoma, did not confirm the clinical benefit of olaratumab in combination with doxorubicin as compared to doxorubicin, a standard-of-care treatment [12]. Continued approval depend on the verification of clinical benefits in a confirmation trial.

In terms of pediatric-type sarcoma, olaratumab, alone and in combination with standard of care, blocks the growth of some preclinical PDGFRa-expressing pediatric bone and soft tissue tumor models [13]. Therapeutic targeting of PDGFRa with small-molecule inhibitors, such as sunitinib and imatinib, has been investigated preclinically and clinically in multiple pediatric cancer types, including osteosarcoma and malignant rhabdoid tumors [14]. Although PDGFRa is not frequently altered in childhood cancers [15], receptor expression is associated with progressive disease and poor prognosis in some pediatric malignancies of bone and soft tissue [16]. Moreover, although PDGFRa expression in cell lines and xenograft models was necessary for olaratumab to demonstrate some antitumor activity in preclinical models, expression alone was insufficient to predict sensitivity [2].

Regarding toxicity profile, despite the retrospective nature of our study, we used the same dosage and dose modification with the phase $\mathrm{lb} / \mathrm{Il}$ clinical trial (patients received olaratumab $[15 \mathrm{mg} / \mathrm{kg}]$ on day 1 and day 8 plus doxorubicin [75 $\mathrm{mg} / \mathrm{m}^{2}$ ] on day 1 of a 21 -day cycles). In our study, toxicities were less frequent than those in the phase $\mathrm{lb} / \mathrm{II}$ clinical trial.

Some limitations of this study include its retrospective nature and small sample size, particularly for histologic subgroup analyses. However, to the best of our knowledge, this is the first study to date to report the efficacy of olaratumab and doxorubicin specifically in an Asian population. In summary, olaratumab plus doxorubicin was safe and effective for patients with advanced STS and should thus be considered as a treatment option for STS patients who have a suitable performance status and adequate heart function.

\section{CONFLICTS OF INTEREST}

No potential conflict of interest relevant to this article was reported.

\section{ACKNOWLEDGMENTS}

This work was supported by a grant from the Korean Health Technology R\&D Project, Ministry of Health and Welfare, Republic of Korea (HI14C2750, HI14C3418).

\section{ORCID}

Joon Young Hur https://orcid.org/0000-0003-0092-8370

Se Hoon Park https://orcid.org/0000-0001-5084-9326

Su Jin Lee https://orcid.org/0000-0003-3405-9935

\section{REFERENCES}

1. Siegel RL, Miller KD, Jemal A. Cancer statistics, 2017. CA Cancer J Clin 2017;67:7-30.

2. Tap WD, Jones RL, Van Tine BA, Chmielowski B, Elias AD, Adkins $D$, et al. Olaratumab and doxorubicin versus doxorubicin alone for treatment of soft-tissue sarcoma: an open-label phase $1 \mathrm{~b}$ and randomised phase 2 trial. Lancet 2016;388:488-97.

3. Coindre JM, Terrier P, Guillou L, Le Doussal V, Collin F, Ranchere $D$, et al. Predictive value of grade for metastasis development in the main histologic types of adult soft tissue sarcomas: a study of 1240 patients from the French Federation of Cancer Centers Sarcoma Group. Cancer 2001;91:1914-26.

4. Davis AM, O'Sullivan B, Turcotte R, Bell R, Catton C, Chabot $P$, et al. Late radiation morbidity following randomization to preoperative versus postoperative radiotherapy in extremity soft tissue sarcoma. Radiother Oncol 2005;75:4853.

5. Judson I, Verweij J, Gelderblom H, Hartmann JT, Schoffski P, Blay JY, et al. Doxorubicin alone versus intensified doxorubicin plus ifosfamide for first-line treatment of advanced or metastatic soft-tissue sarcoma: a randomised controlled phase 3 trial. Lancet Oncol 2014;15:415-23.

6. Schoffski P, Chawla S, Maki RG, Italiano A, Gelderblom H, Choy $\mathrm{E}$, et al. Eribulin versus dacarbazine in previously treated patients with advanced liposarcoma or leiomyosarcoma: a randomised, open-label, multicentre, phase 3 trial. Lancet 2016;387:1629-37.

7. Yoo KH, Kim HS, Lee SJ, Park SH, Kim SJ, Kim SH, et al. Efficacy of pazopanib monotherapy in patients who had been heavily pretreated for metastatic soft tissue sarcoma: a retrospective case series. BMC Cancer 2015;15:154.

8. Reichardt P, Tilgner J, Hohenberger P, Dorken B. Dose-in- 
tensive chemotherapy with ifosfamide, epirubicin, and filgrastim for adult patients with metastatic or locally advanced soft tissue sarcoma: a phase II study. J Clin Oncol 1998;16:1438-43.

9. Shirley M. Olaratumab: first global approval. Drugs 2017; 77:107-12.

10. Antoniou G, Lee ATJ, Huang PH, Jones RL. Olaratumab in soft tissue sarcoma: current status and future perspectives. Eur J Cancer 2018;92:33-9.

11. Tobias A, O'brien MP, Agulnik M. Olaratumab for advanced soft tissue sarcoma. Expert Rev Clin Pharmacol 2017;10:699-705.

12. Olaratumab for STS disappoints in phase III. Cancer Discov 2019;9:312-3.

13. Lowery CD, Blosser W, Dowless M, Knoche S, Stephens J, Li
$\mathrm{H}$, et al. Olaratumab exerts antitumor activity in preclinical models of pediatric bone and soft tissue tumors through inhibition of platelet-derived growth factor receptor $\alpha$. Clin Cancer Res 2018;24:847-57.

14. Wong JP, Todd JR, Finetti MA, McCarthy F, Broncel M, Vyse $S$, et al. Dual targeting of PDGFRa and FGFR1 displays synergistic efficacy in malignant rhabdoid tumors. Cell Rep 2016;17:1265-75.

15. Chmielecki J, Bailey M, He J, Elvin J, Vergilio JA, Ramkissoon S, et al. Genomic profiling of a large set of diverse pediatric cancers identifies known and novel mutations across tumor spectra. Cancer Res 2017;77:509-19.

16. Anderson JL, Denny CT, Tap WD, Federman N. Pediatric sarcomas: translating molecular pathogenesis of disease to novel therapeutic possibilities. Pediatr Res 2012;72:112-21. 